\title{
Grouping oral Candida species by multilocus enzyme electrophoresis
}

\author{
Edvaldo Antonio Ribeiro Rosa, Cássio Vicente Pereira, \\ Rosimeire Takaki Rosa and José Francisco Höfling
}

Microbiology and Immunology Laboratory, Dentistry Faculty of Piracicaba, State University of Campinas, Av. Limeira 901, CEP 13414-900, CP 52, Piracicaba, SP, Brazil
Author for correspondence: José Francisco Höfling. Tel: +55 19430 5321. Fax: +55 194305018. e-mail: hofling@fop.unicamp.br

\begin{abstract}
Multilocus enzyme electrophoresis (MLEE) and numerical taxonomic methods were used to establish the degrees of relatedness among five Candida species commonly isolated from humans oral cavities. Of twenty enzymic systems assayed, five showed no enzymic activity (aspartate dehydrogenase, mannitol dehydrogenase, sorbitol dehydrogenase, glucosyl transferase and $\alpha$-amylase). The obtained data revealed that some of these enzymes are capable of distinguishing strains of different species, but most of them could not organize all strains in their respective species-specific clusters. Numerical classification based on MLEE polymorphism must be regarded for surveys involving just one Candida species.
\end{abstract}

Keywords: oral Candida spp., cluster analysis, MLEE

\section{INTRODUCTION}

Candida species, particularly Candida albicans, remain the most common fungi found in the oral cavity of humans and, in recent years, have received attention because of their involvement in a number of cases of opportunist oral infections in patients with AIDS and those having immunosuppressive medication. Of epidemiological interest, characterization procedures that produce molecular fingerprints have been used to establish possible relationships among Candida isolates that could have some oral relevance (McCullough et al., 1996). Multilocus enzyme electrophoresis (MLEE) is a resource that has been used in studies involving a large number of micro-organisms (Selander \& Levin, 1980; Soltis et al., 1980; Okunishi et al., 1979) including Candida species (Lehmann et al., 1989a; Pujol et al., 1993; Reynes et al., 1996). These studies have highlighted the usefulness of some enzymic systems in discriminating less related strains or even species from other genera. The purpose of the

\footnotetext{
Abbreviations: ACO, aconitase; $\mathrm{ADH}$, alcohol dehydrogenase; $\alpha$-AM, $\alpha$ amylase; ASDH, aspartate dehydrogenase; CAT, catalase; $\alpha$-EST, $\alpha$-esterase; $\beta$-EST, $\beta$-esterase; G6PDH, glucose-6-phosphate dehydrogenase; GDH, glucose dehydrogenase; GOT, glutamate-oxaloacetate transaminase; GTF, glucosyl transferase; IDH, isocitrate dehydrogenase; LAP, leucine aminopeptidase; $\mathrm{LDH}$, lactate dehydrogenase; $\mathrm{MADH}$, mannitol dehydrogenase; $\mathrm{MDH}$, malate dehydrogenase; ME, malic enzyme; MLEE, multilocus enzyme electrophoresis; OTU, operational taxonomic unit; PO, peroxidase; SDH, sorbitol dehydrogenase; SOD, superoxide dismutase; UPGMA, unweighted pair group method with arithmetic averages.
}

present study is to evaluate the parity existing among some enzymic systems for fingerprinting five Candida species (C. albicans, Candida tropicalis, Candida krusei, Candida parapsilosis and Candida guilliermondii) isolated from the saliva of healthy human subjects.

\section{METHODS}

Candida strains. Representative strains of different Candida species isolated from oral cavities and identified by colony characteristics on CHROMagar Candida differential medium (Anson \& Allen, 1997; Bernal et al., 1996; San Milan et al., 1996), chlamydospore and germ tube formation, and by sugar fermentation and assimilation (Sandven, 1990), were obtained from the Microbiology and Immunology Laboratory, Dentistry College of São José dos Campos: $C$. albicans $\left(97 \mathrm{a}, \mathrm{F} 72, \mathrm{E} 37,17 \mathrm{~b}, \mathrm{CBS} 562^{\mathrm{T}}\right)$; C. guilliermondii (FCF405, FCF152, CBS 566 $6^{\mathrm{T}}$ ); C. parapsilosis (21c, 7a, CBS $\left.604^{\mathrm{T}}\right) ;$ C. krusei $\left(1 \mathrm{M} 90,4 \mathrm{c}, \mathrm{CBS} 573^{\mathrm{T}}\right)$; and C. tropicalis (1b, FCF430, CBS 94 $4^{\mathrm{T}}$ ). All strains (excluding the type strains) were isolated from the oral cavities of healthy subjects. In this work, the type strain of Saccharomyces cerevisiae (CBS $1171^{\mathrm{T}}$ ) was used as an extra-generic organism.

Cell cultivation and enzyme extraction. All the strains were grown overnight in $50 \mathrm{ml} \mathrm{YPD}$ medium (2\% dextrose, $2 \%$ peptone, $1 \%$ yeast extract), on a shaker table at 150 r.p.m. and $30{ }^{\circ} \mathrm{C}$. Cells were harvested by centrifugation of total culture medium volume at $2000 \mathrm{~g}$ for $3 \mathrm{~min}$ and pellets were washed four times with cold sterile water to ensure complete removal of traces of culture medium and extracellular metabolites (Woontner \& Jaehning, 1990). The washed pellets were transferred to $2 \mathrm{ml}$ microcentrifuge tubes and equal amounts of acid-washed glass beads and $200 \mu \mathrm{l}$ cold 
sterile water were added. The tubes were placed in a MiniBead Beater cell disrupter (Biospec) and cell lysis was carried out at 4600 r.p.m. for four $30 \mathrm{~s}$ bursts, at intervals of $5 \mathrm{~min}$, in which the samples were conditioned in an ice bath. After cell disruption, the microcentrifuge tubes were centrifuged at $10000 \mathrm{~g}$ for $2 \mathrm{~min}$, and the supernatants were applied to Whatman 3 filter paper wicks of $5 \times 12 \mathrm{~mm}$. These wicks were maintained at $-70^{\circ} \mathrm{C}$.

Starch gel electrophoresis. Electrophoresis was carried out according to Val et al. (1981); gels were formed by solubilizing hydrolysed corn starch Penetrose 30 (Refinações de Milho Brasil, São Paulo) at a final concentration of $13 \%$ in diluted 1:30 Tris-citrate buffer $\mathrm{pH} 8 \cdot 0$ and vigorously agitated heating over a Bunsen burner. The formed gels were poured in perplex casting moulds $(200 \times 120 \times 10 \mathrm{~mm})$ and left on the bench, at room temperature, until complete solidification. They were cut longitudinally $2.5 \mathrm{~cm}$ from one border. The $2.5 \mathrm{~cm}$ segments were separated and the wicks were applied on the cut. Wicks with $0 \cdot 2 \%$ bromophenol blue were applied to both extremities of the cuts to indicate migration. After joining the parts, cotton cloth bridges were made to connect the gels to electrode tanks containing Tris-citrate buffer $\mathrm{pH} \mathrm{8.0} \mathrm{(Selander} \mathrm{et} \mathrm{al.,}$ 1986; Caugant \& Sandven, 1993). Electrophoresis was carried out at $4{ }^{\circ} \mathrm{C}$ and $130 \mathrm{~V}$ until the migration markers had moved at least $80 \mathrm{~mm}$ from the application point. At this time, electrophoresis was terminated and the gels, placed horizontally, were sliced horizontally into $1.2 \mathrm{~mm}$ thickness using a small device in which a thin fishing cord sliced the gels over an elevatory table.

Band revelation. The gel slices were stained to reveal the active enzyme bands, according to Selander et al. (1986) protocols. Enzymic systems assayed were: alcohol dehydrogenase (ADH; EC 1.1.1.1); lactate dehydrogenase (LDH; EC 1.1.1.27); malate dehydrogenase (MDH; EC 1.1.1.37); isocitrate dehydrogenase (IDH; EC 1.1.1.42); glucose-6phosphate dehydrogenase (G6PDH; EC 1.1.1.49); aspartate dehydrogenase (ASDH; EC 1.4.3.x); glucose dehydrogenase (GDH; EC 1.1.1.47); mannitol dehydrogenase (MADH; EC 1.1.1.67); sorbitol dehydrogenase (SDH; EC 1.1.1.14); malic enzyme (ME; EC 1.1.1.40); aconitase (ACO; EC 4.2.1.3); catalase (CAT; EC 1.11.1.6); superoxide dismutase (SOD; EC 1.15.1.1); glutamate-oxaloacetate transaminase (GOT; EC 2.6.1.1); $\alpha$-esterase (EST; EC 3.1.1.1); $\beta$-esterase (EST; EC 3.1.1.1); leucine aminopeptidase (LAP; EC 3.4.1.1); glucosyl transferase (GTF; EC 2.4.1.11); peroxidase (PO; EC 1.11.1.7); and $\alpha$-amylase ( $\alpha$-AM; EC 3.2.1.1).

Computing numerical data. Dendrograms were generated for the different enzymic systems by using the same measurement of relatedness, the Simple Matching association coefficient $\left(S_{\mathrm{SM}}\right.$; Sokal \& Michener, 1958; Sneath \& Sokal, 1973), based on band positions computed with the NTSYS software package, version 1.70 (Applied Biostatistics) $S_{\mathrm{SM}}$ measures the proportion of bands with the same and the different relative mobility values in patterns of two operational taxonomic units (OTU), $\mathbf{k}$ and $\mathbf{j}$, by the formula: $S_{\mathrm{SM}}=E /(E+b+c)$, where $E$ is the positive combination of bands (present and absent) shared by OTUs $\mathbf{k}$ and $\mathbf{j}, b$ is the number of bands unique to OTU $\mathbf{k}$, and $c$ is the number of bands unique to OTU $\mathbf{j}$. In the present study, an $S_{\mathrm{SM}}$ value of 1.00 represents identical matches (i.e. all the bands in the patterns of OTUs $\mathbf{k}$ and $\mathbf{j}$ match), an $S_{\mathrm{SM}}$ value of 0.00 represents no matches, and $S_{\mathrm{SM}}$ values of $0.01-0.99$ represent increasing proportions of matched bands. Dendrograms, represented by non-rooted trees, based on $S_{\mathrm{SM}}$ values were generated by the unweighted pair group method with arithmetic means (UPGMA; Rohlf, 1963; Sneath \& Sokal, 1973).

\section{RESULTS}

The one-dimensional electrophoreses of protein extracts of 12 Candida strains, their respective type strains and the $S$. cerevisiae type strain, showed that, of twenty assayed enzymes, five (ASDH, MADH, SDH, GTF and $\alpha$-AM) did not show any enzymic activity. The remaining systems provided electrophoretic bands that enabled the fifteen individual dendrograms shown in Fig. 1 to be constructed. C. albicans strains CBS $562^{\mathrm{T}}, 97 \mathrm{a}, \mathrm{F} 72$, and E37 clustered in dendrograms A (ADH), B (ME), E (IDH), F (LDH), H (ACO) and K (CAT). C. albicans strain $17 \mathrm{~b}$ showed atypical MLEE patterns for all assayed enzymes, grouping with strains of other Candida species in a non-repetitive way. The two clinical strains of $C$. guilliermondii (FCF152 and FCF405) only clustered together in the dendrogram derived from the IDH system. Dendrogram G (MDH) showed grouping of these clinical strains of $C$. guilliermondii with the inclusion of $C$. albicans strain E37; the type strain of $C$. guilliermondii clustered with three $C$. albicans strains (CBS 562 ${ }^{\mathrm{T}}, 97 \mathrm{a}$, and F72). For C. krusei, two strains, CBS $573^{\mathrm{T}}$ and $1 \mathrm{M} 90$, were grouped by the IDH system. The CAT system could group strains CBS $573^{\mathrm{T}}$ and $4 \mathrm{c}$, and the PO system formed a composite cluster with the inclusion of the atypical C. albicans strain $17 \mathrm{~b}$ between $C$. krusei strains $1 \mathrm{M} 90$ and 4c. Among $C$. tropicalis strains, the type strain $\left(\mathrm{CBS} 94^{\mathrm{T}}\right)$ and the clinical isolate FCF430 were the specimens that revealed the closest relationship $\left(S_{\mathrm{SM}}=1.00\right)$ for four enzymic systems (IDH, LDH, $\alpha$-EST and $\beta$-EST); for the ADH, ME and MDH systems, these strains grouped with $S_{\mathrm{SM}}>0 \cdot 85$. All three strains of $C$. parapsilosis appeared together in a single cluster for the dendrograms of G6PDH, IDH, LDH, ACO, CAT, GOT, LAP and SOD although in the four latter trees, they were associated with strains of other species. The $S$. cerevisiae type strain CBS $1171^{\mathrm{T}}$ combined with different species of Candida depending on the enzymic system.

The results of the individual MLEE analyses were pooled for each strain and a non-rooted relatedness dendrogram of the 18 analysed strains, based on the similarity calculated by $S_{\mathrm{SM}}$ values, was constructed (Fig. 2). Nine phenons (clusters) were established by the perpendicular line (dashed) that represents the average value for $S_{\mathrm{SM}}$ of all OTUs (i.e. 0.841).

Phenon I contained the strains CBS 562 ${ }^{\mathrm{T}}, 97 \mathrm{a}, \mathrm{F} 72$ and E37 (C. albicans), grouped with $S_{\mathrm{SM}} \geqslant 0.898$. Phenon II contained two strains of $C$. guilliermondii (FCF152 and FCF405) and one of C. tropicalis (1b) as components, grouped with $S_{\mathrm{SM}} \geqslant 0 \cdot 847$. Phenon III just contained CBS $1171^{\mathrm{T}}$, the type strain of $S$. cerevisiae. Phenon IV contained the three strains of $C$. parapsilosis (CBS $604^{\mathrm{T}}, 21 \mathrm{c}$ and $7 \mathrm{a}$ ) and the atypical $C$. albicans strain $17 \mathrm{~b}$, with $S_{\mathrm{SM}} \geqslant 0.845$. Phenon V is a cluster composed of a unique C. krusei strain (4c). Phenon VI 
Oral Candida species grouped by MLEE

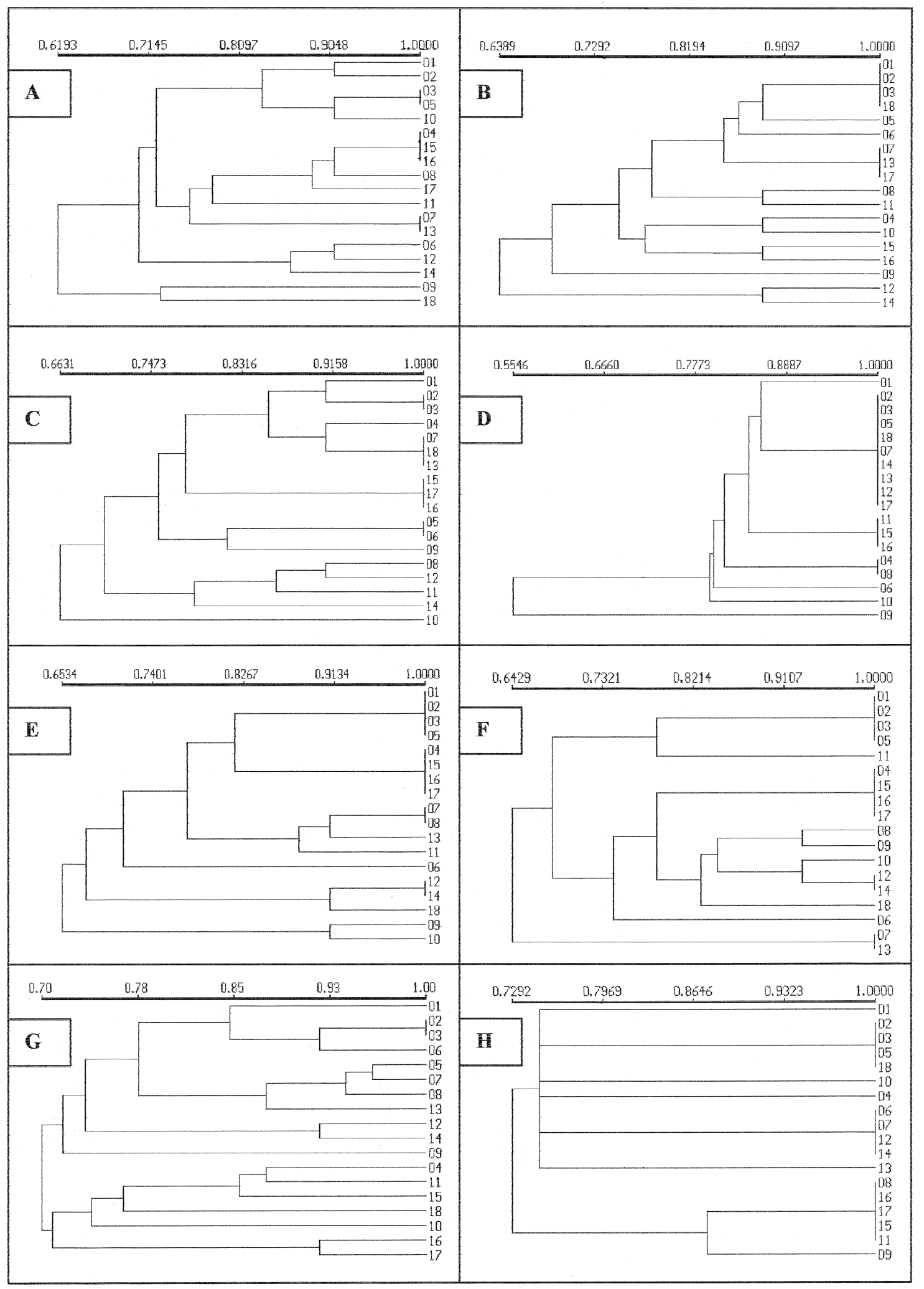

Fig. 1. For legend see page 1346. 


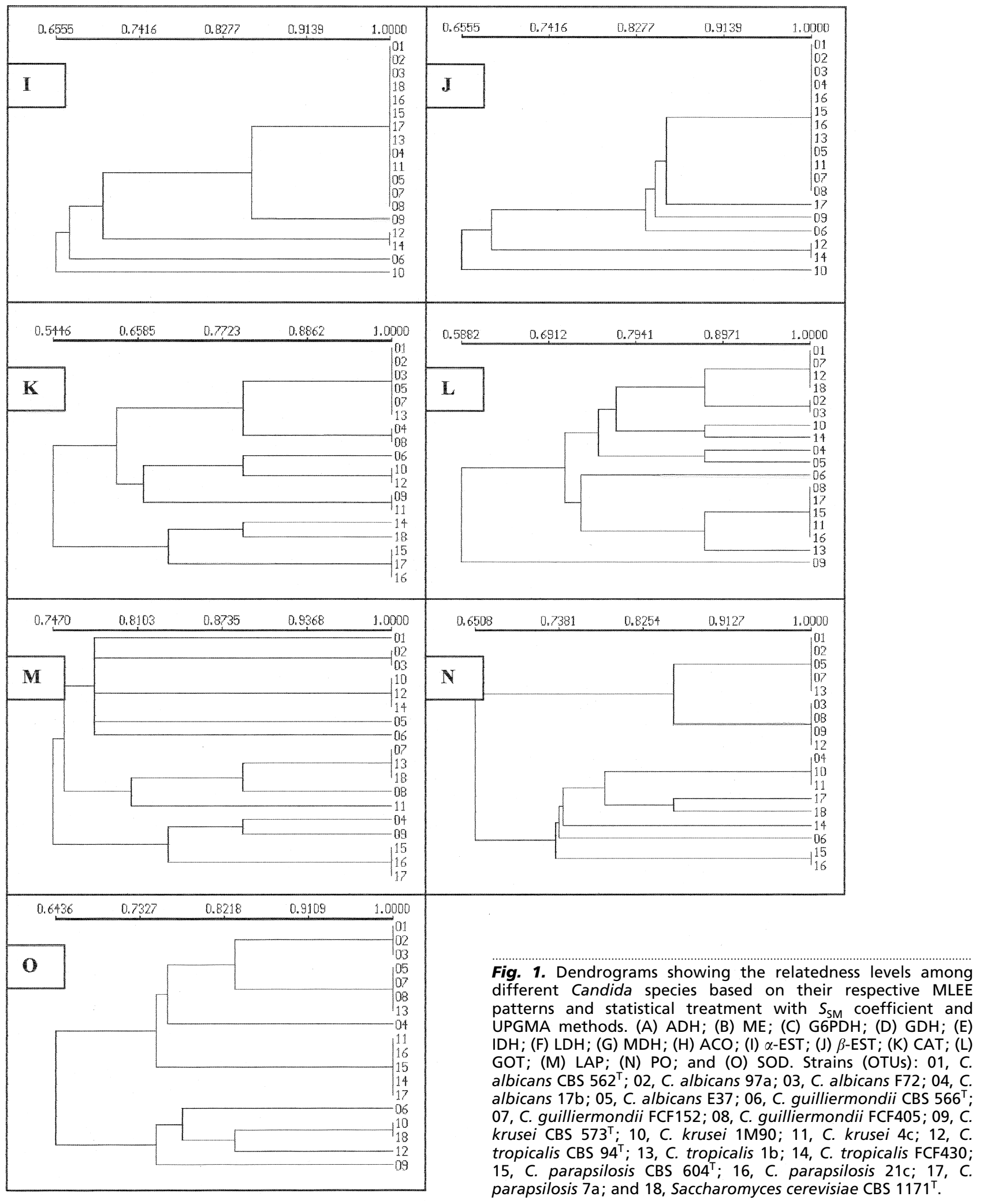




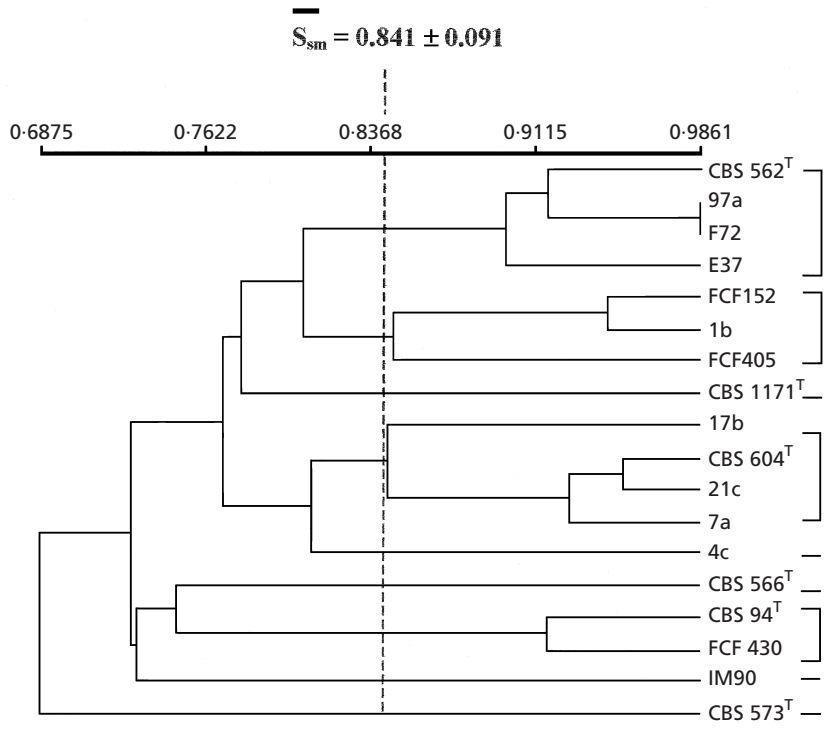

Phenon I

Phenon II

Phenon III

Phenon IV

Phenon V

Phenon VI

Phenon VII

Phenon VIII

Phenon IX
Fig. 2. Non-rooted relatedness dendrogram of oral Candida species grouped by the sum of all enzymic patterns, $S_{S M}$ coefficient and UPGMA algorithm. contained CBS 566 ${ }^{\mathrm{T}}$, the type strain of $C$. guilliermondii. Phenon VII is composed of two strains of $C$. tropicalis (CBS 94 ${ }^{\mathrm{T}}$ and FCF430) grouped with $S_{\mathrm{SM}}=0.917$. Phenon VIII has strain 1 M90 of $C$. krusei. Phenon IX contained the type strain of C. krusei (CBS $\left.573^{\mathrm{T}}\right)$.

\section{DISCUSSION}

Yeasts of the genus Candida form a heterogeneous group containing species whose teleomorph states are in the Ascomycota or those that do not have a defined perfect state. Several species of Candida can be isolated from the oral cavity, justifying the necessity for understanding their ecological involvement. Reports based on MLEE patterns of Candida have supplied useful information in oral epidemiological surveys (Reynes et al., 1996; Pujol et al., 1993). Among the wide range of enzyme classes, the dehydrogenases, hydrolases and transferases, as well as some others, are the most interesting enzymes applicable to the MLEE technique, due to their relative stability, substrate specificity and occurrence in living organisms (Dixon \& Webb, 1979; Selander et al., 1986; Gabriel \& Gersten, 1992).

In Fig. 1, the enzymic system that could group the majority of strains in their respective species clusters was IDH. Such a fact has already been pointed out by Lehmann et al. (1989a), who also noted that IDH and SDH solely distinguish species and do not have any value in biotyping Candida isolates. In the present investigation, for different repetitions of SDH band detection protocols, such band patterns could not be obtained, even when other protocols were tested. However, the systems that gave the worst groupings were GDH, $\alpha$-EST and $\beta$-EST, possibly due to the non-formation of bands in many strains. $C$. parapsilosis strains could be grouped together with $S_{\mathrm{SM}}$ values of $1 \cdot 000$, in species-specific or composite clusters, in most of the non-dehydrogenases (ACO, CAT, GOT, LAP and SOD), showing this to be the species whose strains are the most related, even being isolated from different individuals. The same behaviour was detected for $C$. albicans strains CBS 562 ${ }^{\mathrm{T}}, 97 \mathrm{a}$ and F72, in different enzymic systems.

The non-rooted dendrogram presented in Fig. 2 shows the sum of all partial dendrograms in Fig. 1. Clusters were formed from the limiting line derived from the average of all OTU similarities of $S_{\mathrm{SM}}=0.841$ (SD of 0.091). Some strains clustered together either with others of their own species (species-specific clusters) as in phenons I ( $C$. albicans) and VII ( $C$. tropicalis) or with different species (composite clusters) as phenons II and IV.

According to Fig. 2, the MLEE technique could group most $C$. albicans strains in a single phenon, with exception of strain $17 \mathrm{~b}$ which was shown to be less related. This fact was also observed in previous assays involving SDS-PAGE of whole-cell proteins for these strains (Höfling et al., 1999). Strain 17b was reidentified in order to determine whether or not it is a $C$. albicans isolate. The phenotypic characteristics, including the use of CHROMagar Candida medium which differentiates $C$. albicans from the recently described Candida dubliniensis (Schoofs et al., 1997), confirmed that this isolate was indeed a $C$. albicans strain. The analysed enzymes also grouped all strains of $C$. parapsilosis with $C$. albicans strain $17 \mathrm{~b}$. This aspect of composite cluster generated by MLEE has been previously observed by other authors. Smith et al. (1990), characterizing different species of Brettanomyces and Dekkera, obtained a phenogram in which some strains could not be grouped with high similarity values in their respective species-specific clusters and with interference of some strains in other clusters. Jones \& Noble (1982) established electrophoretic 
comparisons among species of dermatophytes based on the MLEE technique and obtained a dendrogram in which isolates from certain species were included in the inner taxa of other species or even of other genera. These authors pointed out that this may occur when only a few isolates of each species are included in the surveys. Boerlin et al. (1995) used 16 enzymic systems for characterizing 21 genetically atypical strains of chlamydospore-forming, germ-tube-positive C. albicans recovered from human immunodeficiency viruspositive drug users, and demonstrated that some of these strains grouped in different clusters, showing high diversity in allelic composition.

Extensive enzyme heterogeneity among strains of Candida or other yeast genera has already been observed by other groups of researchers who pointed out that it may increase the possibility of dividing such specimens in various groups or clusters (Lehmann et al., 1989a, b, 1993; Caugant \& Sandven, 1993; Naumov et al., 1997). Lehmann et al. (1991) related the phenomenon of isoenzymic patterns of C.albicans changing during its conservation in laboratories, which could increase the apparent polymorphism. Pujol et al. (1997) found atypical strains of C. albicans in AIDS patients, showing diverse allelic polymorphism. The same investigators included some strains of $C$. tropicalis, Candida glabrata and C. krusei in the survey, obtaining characteristic species-specific clusters. However, the fact that just a few specimens of these species were added could have influenced the organization of such clusters.

In order to ensure that the UPGMA algorithm fits the assemblance between two OTUs in the dendrogram construction well, a product-moment correlation coefficient $\left(r_{\mathrm{CS}}\right)$ was computed between the elements $S_{\text {JK }}$ of the original similarity matrix $S$ and co-phenetic values $C_{\mathrm{JK}}$ of the matrix $C$ derived from the dendrogram. This co-phenetic correlation coefficient is a measure of the agreement between similarity values implied by the dendrogram and those of the original similarity matrix (Sokal \& Rohlf, 1962). This coefficient has a value of 0.932 , which is considered good according to Sneath \& Sokal (1973), i.e. it is between 0.60 and 0.95 , and Sokal \& Rohlf (1970), i.e. it is higher than 0.90 ; this value supports the results of Farris (1969), which pointed out that the UPGMA algorithm will always maximize $r_{\mathrm{CS}}$ values.

All the strains employed here were previously and independently classified up to species level in two different laboratories by mean chlamydospore and germ tube formation, and fermentation and assimilation of sugars; the same results were obtained. This evidence discards the possibility of poor identification of the strains in previous studies.

Based on the presented observations, it is proposed that the grouping of Candida species by mean MLEE patterns from assayed enzymes followed by numerical taxonomy statistical treatment is not efficient when involving few isolates from more than one species. Such a resource should be utilized for surveys conducted with a single species of Candida, in which the MLEE technique has already proved to be a method useful for systematic or epidemiological purposes.

\section{ACKNOWLEDGEMENTS}

The authors are indebted to 'Fundação de Amparo à Pesquisa do Estado de São Paulo' and 'Fundo de Apoio ao Ensino e Pesquisa-UNICAMP for the financial support given to this work.

\section{REFERENCES}

Anson, J. J. \& Allen, K. D. (1997). Evaluation of CHROMagar Candida medium for the isolation and direct identification of yeast species from the female genital tract. Br J Biomed Sci 54, 237-239.

Bernal, S., Martin Mazuelos, E., Garcia, M., Aller, A. I., Martinez, M. A. \& Gutierrez, M. J. (1996). Evaluation of CHROMagar Candida medium for the isolation and presumptive identification of Candida of clinical importance. Diagn Microbiol Infect Dis 24, 201-204.

Boerlin, P., Boerlin-Petzold, F., Durussel, C., Addo, M., Pagani, J. L., Chave, J. P. \& Bille, J. (1995). Cluster of oral atypical Candida albicans isolates in a group of human immunodeficiency viruspositive drug users. J Clin Microbiol 33, 1129-1135.

Caugant, D. A. \& Sandven, A. (1993). Epidemiological analysis of Candida albicans strains by multilocus enzyme electrophoresis. $J$ Clin Microbiol 31, 215-220.

Dixon, M. \& Webb, E. C. (1979). Enzymes. 3rd edn. New York: Academic Press.

Farris, J. S. (1969). On the cophenetic correlation coefficient. Syst Zool 18, 279-285.

Gabriel, O. \& Gersten, D. M. (1992). Staining for enzymatic activity after gel electrophoresis. Anal Biochem 203, 1-21.

Höfling, J. F., Campos, A. S., Pereira, C. V., Rosa, R. T. \& Rosa, E. A. R. (1999). Preliminary characterization and grouping of Candida species by numerical analysis of protein profiles obtained by polyacrylamide gel electrophoresis. Rev Iberoam Micol 16, 27-29.

Jones, M. G. \& Noble, W. C. (1982). An electrophoretic study of enzymes as a tool in the taxonomy of the dermatophytes. J Gen Microbiol 120, 1101-1107.

Lehmann, P. F., Hsiao, C. B. \& Salkin, I. F. (1989a). Proteins and electrophoresis profiles of selected Candida species. J Clin Microbiol 27, 400-404.

Lehmann, P. F., Kemker, B. J., Hsiao, C. B. \& Dev, S. (1989b). Isoenzyme biotypes of Candida species. J Clin Microbiol 27, 2514-2521.

Lehmann, P. F., Wu, L. C. \& Mackenzie, D. W. (1991). Isoenzyme changes in Candida albicans during domestication. J Clin Microbiol 29, 2623-2625.

Lehmann, P. F., Wu, L. C., Pruitt, W. R., Meyer, S. A. \& Ahearn, D. G. (1993). Unrelatedness of groups of yeasts within the Candida haemulonii complex. J Clin Microbiol 31, 1683-1687.

McCullough, M. J., Ross, B. C. \& Reade, P. C. (1996). Candida albicans: a review of its history, taxonomy, virulence attributes, and methods of strain differentiation. Int $J$ Oral Maxillofac Surg 25, 136-144. 
Naumov, G. I., Naumova, E. S. \& Sniegowisk, P. D. (1997). Differentiation of European and far east Asian populations of Saccharomyces paradoxus by allozyme analysis. Int $J$ Syst Bacteriol 47, 341-344.

Okunishi, M., Yamada, K. \& Komagata, K. (1979). Electrophoretic comparison of enzymes from basidiomycetes in different stages of development. J Gen Appl Microbiol 25, 329-334.

Pujol, C., Reynes, J., Renaud, F., Raymond, M., Tibayrenc, M., Ayala, M. J., Janbon, F., Mallie, M. \& Bastide, J. M. (1993). The yeast Candida albicans has a clonal mode of reproduction in a population of infected human immunodeficiency virus-positive patients. Proc Natl Acad Sci USA 90, 9456-9459.

Pujol, C., Renaud, F., Mallie, M., de Meeus, T. \& Bastide, J. M. (1997). Atypical strains of Candida albicans recovered from AIDS patients. J Med Vet Mycol 35, 115-121.

Reynes, J., Pujol, C., Moreau, C., Mallie, M., Renaud, F., Janbon, F. \& Bastide, J. M. (1996). Simultaneous carriage of Candida albicans strains from HIV-infected patients with oral candidiasis: multilocus enzyme electrophoresis analysis. FEMS Microbiol Lett 137, 269-273.

Rohlf, F. J. (1963). Classification of Aedes by numerical taxonomic methods (Diptera: Culicidae). Ann Entomol Soc Am 56, 798-804.

Sandven, P. (1990). Laboratory identification and sensitivity testing yeast isolates. Acta Odontol Scand 48, 27-36.

San Millan, R., Ribacoba, L., Ponton, J. \& Quindos, G. (1996). Evaluation of a commercial medium for identification of Candida species. Eur J Clin Microbiol Infect Dis 15, 153-158.

Schoofs, A., Odds, F. C., Colebunders, R., Leven, M. \& Goosens, H. (1997). Use of specialised isolation media for recognition and identification of Candida dubliniensis isolates from HIV-infected patients. Eur J Clin Microbiol Infect Dis 16, 296-300.

Selander, R. K. \& Levin, B. R. (1980). Genetic diversity and structure in Escherichia coli populations. Science 210, 545-547.

Selander, R. K., Caugant, D. A., Ochman, H., Musser, J. M., Gilmour, M. N. \& Whittam, T. S. (1986). Methods of multilocus enzyme electrophoresis for bacterial population genetics and systematics. Appl Environ Microbiol 51, 873-884.

Smith, M. T., Yamazaki, M. \& Poot, G. A. (1990). Dekkera, Brettanomyces and Eeniella: electrophoretic comparison of enzymes and DNA-DNA homology. Yeast 6, 299-310.

Sneath, P. H. A. \& Sokal, R. Q. (1973). Numerical Taxonomy. San Francisco: Freeman.

Sokal, R. R. \& Michener, C. D. (1958). A statistical method for evaluating systematic relationships. Univ Kans Sci Bull 38, 1409-1438.

Sokal, R. R. \& Rohlf, F. J. (1962). The comparison of dendrograms by objective methods. Taxon 11, 33-40.

Sokal, R. R. \& Rohlf, F. J. (1970). The intelligent ignoramus, an experiment in numerical taxonomy. Taxon 19, 305-319.

Soltis, D. E., Haufler, C. H. \& Gastony, G. J. (1980). Detecting enzyme variation in the fern genus Bommeria: an analysis of methodology. Syst Bot 5, 30-38.

Val, A. L., Schwantes, A. R., Schwantes, M. L. B. \& De Luca, P. H. (1981). Amido hidrolisado de milho como suporte eletroforético. Cienc Cult 33, 992-996.

Woontner, M. \& Jaehning, J. A. (1990). Accurate initiation by RNA polymerase II in a whole cell extract from Saccharomyces cerevisiae. J Biol Chem 265, 8979-8982. 